\title{
¿Son los rincones una buena estrategia metodológica para la enseñanza del Entorno en Educación Infantil?
}

FÁTIMA ROdRÍGUEZ-MARÍN

Universidad de Sevilla

Departamento de Didáctica de las

Ciencias Experimentales y Sociales

frodmar@us.es

ORCID: https://orcid.org/0000-0003-0771-6944

D.O.I.: $\mathrm{http://dx.doi.org/10.12795/JDU.2018.i01.98}$

Pp.: 1741-1755

\section{Resumen}

En la siguiente comunicación se presenta una propuesta de intervención para trabajar una de las estrategias metodológicas que se consideran fundamentales en la Educación Infantil: Los rincones. Dicha propuesta se ha llevado a cabo en la asignatura "Enseñanza del Entorno Natural en la etapa de 0-6 años" de 30 curso del Grado de Educación Infantil de forma coordinada con la asignatura de Conocimiento del Entorno Social. El modelo didáctico personal seguido para el desarrollo de esta experiencia, se basa fundamentalmente en el modelo de Investigación en la Escuela, ya que considero que dicho modelo recoge los principios didácticos que quiero trabajar con mi alumnado. 
Palabras clave: Enseñanza del Entorno Natural en la etapa de 0 a 6 años, Educación Infantil, docencia universitaria, experimentación docente universitaria, rincones.

\section{Descripción contexto}

La experimentación docente se ha realizado en el marco de la asignatura cuatrimestral "Enseñanza del Entorno Natural en la etapa de 0 a 6 años" de 3o curso del Grado de Educación Infantil. La asignatura se imparte durante 4 horas a la semana, y se trabaja de forma coordinada con la asignatura Conocimiento del Entorno Social contando por tanto con 8 horas a la semana. La intervención se realizada en el grupo 1, el cual está formado por 75 personas matriculadas, asistiendo regularmente 65 personas (siendo 61 alumnas y 1 alumno). La asistencia a clase es bastante alta debido a que la mayoría del alumnado elige la opción presencial, en el que es necesario asistir al $80 \%$ de las clases, y la organización de la clase es en grupos de trabajo de 4-5 personas.

\section{Diseño previo del Ciclo de Mejora Docente}

En este ciclo de mejora se ha seleccionado un elemento que se trabaja de manera transversal: los rincones. Se ha determinado un problema central que da sentido a todo el trabajo que llevaremos a cabo "¿Son los rincones una buena estrategia metodológica para la Enseñanza del Entorno en Educación Infantil?", de esta pregunta, derivan tres subproblemas que nos permitirán ir abordando los diferentes contenidos que hemos considerado esenciales. Como se puede apreciar en la figura 1, de alguno de estos subproblemas derivan otros que creemos de segundo nivel y que aparecen con una letra algo más pequeña y con un color más claro. A través de flechas hemos señalado las 
relaciones que existen entre los contenidos. Así por ejemplo hemos empleado flechas de color rojo para marcar la relación entre las cuestiones claves y flechas bidireccionales para señalar la relación bidireccional entre algunos de los contenidos recogidos. Hemos usado también un código de color para así diferenciar los contenidos de carácter conceptual (rojo), de los que tienen carácter procedimental (verde) y de los de carácter actitudinal, que hemos considerado transversales para todos los bloques (círculos morados).

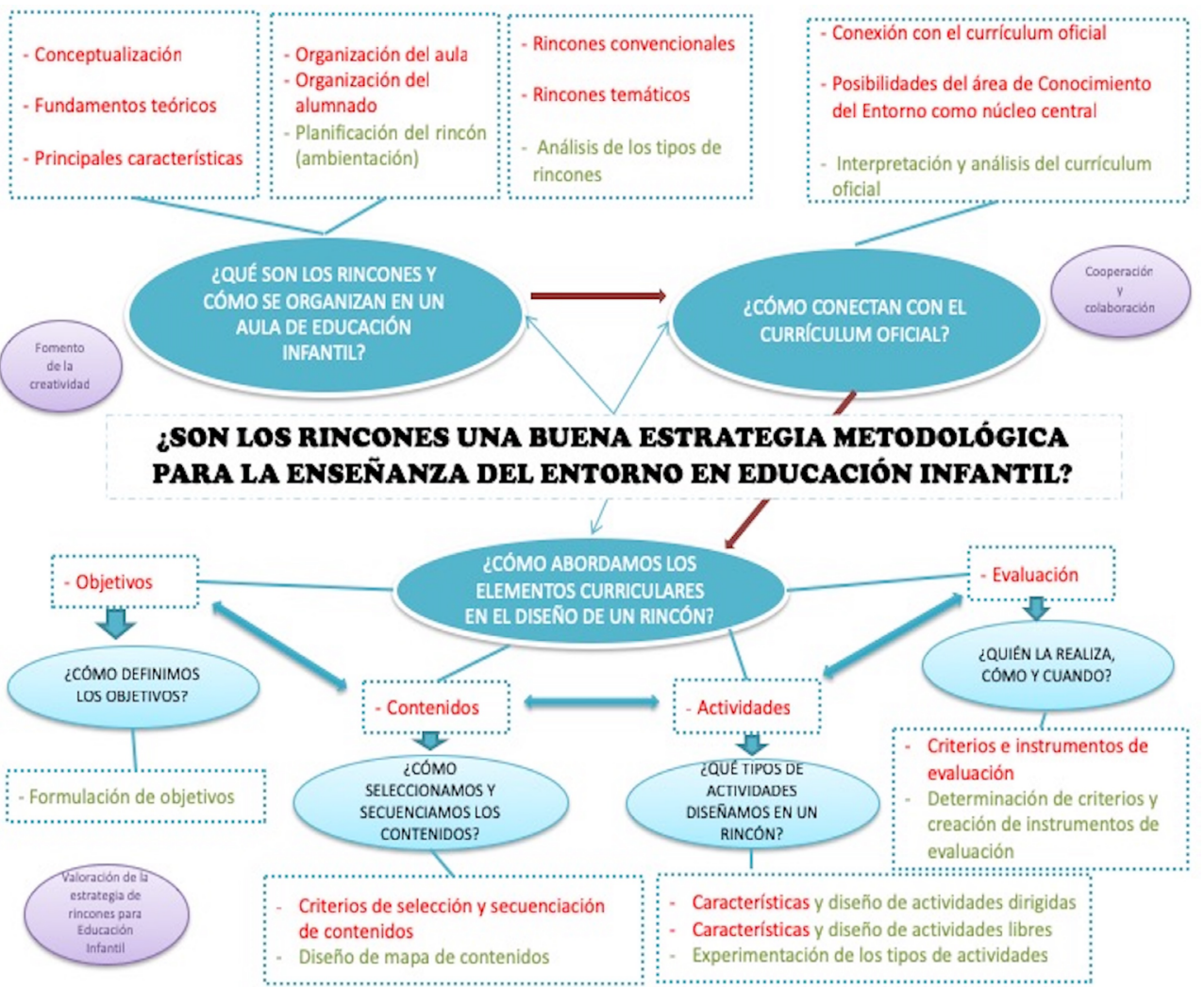

Figura 1. Mapa de contenidos-problemas Inicial

Jornadas de Formación e Innovación Docente del Profesorado | № 1 (2018)

cC (i) $\Theta$ Esta obra se distribuye con la licencia Creative Commons Reconocimiento-NoComercial-SinObraDerivada $\quad 4.0$ Internacional (CC BY-NC-ND 4.0.) 


\section{Modelo metodológico y Secuencia de actividades}

Los elementos que conforman mi Modelo Didáctico Personal (Ballenilla, 2003) son de diverso tipo y origen, siendo mi referente, un modelo basado en la investigación del alumno (García, 2004). Pese a que este modelo no está constituido por una secuencia fija de pasos, sí se pueden distinguir pautas necesarias y comunes que pueden ser concretadas en ciclos metodológicos (García, 2004), y que fue la propuesta de trabajo seguida para poner en marcha y analizar el proceso que he llevado a cabo:

a) Delimitación del problema: El punto de partida ha sido la formulación de un problema que el alumnado debe resolver, considerando que tiene más sentido formular problemas que conecten con el alumnado y, a la vez, exijan el conocimiento que queremos enseñar para su resolución.

b) Primer tratamiento desde las ideas de los alumnos/as: El segundo paso que realicé fue conocer y tener en consideración cuáles son las idas previas que los alumnos tienen sobre el problema que le hemos planteado, a través de un cuestionario.

c) Aporte de información nueva y contraste de información: Antes de este paso, se realizó una actividad de motivación. Posteriormente se ha ido aportando información utilizando fuentes lo más diversas posibles (videos, lecturas, análisis de materiales, trabajo en grupo,...), que entren en confrontación con las ideas previas - hipótesis que el alumnado planteó en la etapa anterior.

d) Recapitulación: Resultado de la confrontación anterior esperaba la reformulación de dichas ideas llegando a conclusiones plausibles.

e) Síntesis y comunicación de lo aprendido: Para ello el alumnado debe llevar a cabo una labor de sintesis de lo aprendido, para lo que se solicitó 
un trabajo final. Además para cerrar este proceso se plantea la realización de una puesta en común en gran grupo presentando los rincones.

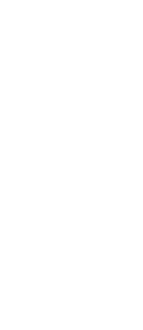

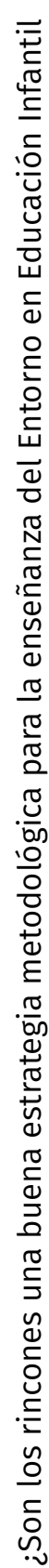

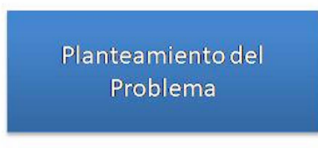

IDEAS DE LOS

ALUMNOS

INICIAL
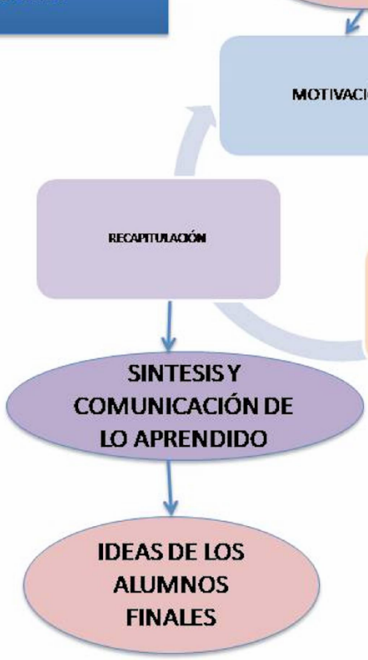

Figura 2. Modelo Didáctico Personal

A continuación se presenta la secuencia de actividades diseñada:

Tabla 1.

\begin{tabular}{|c|l|}
\hline \multirow{2}{*}{ IDEAS INICIALES } & $\begin{array}{l}\text { Sesión 1: ¿Qué son los rincones y cómo se organizan en un } \\
\text { aula de Educación Infantil? } \\
\text { Presentación del bloque. } \\
\text { Organización de los grupos de trabajo. } \\
\text { Cuestionario de ideas iniciales. } \\
\text { Puesta en común de las respuestas ofrecidas en los cues- } \\
\text { tionarios iniciales. }\end{array}$ \\
\hline AMPLIACIÓN Y & $\begin{array}{l}\text { Lectura de documentos seleccionado por las docentes so- } \\
\text { bre los rincones en pequeño grupo extrayendo las ideas } \\
\text { CONincipales. } \\
\text { Puesta en común entre los grupos que hayan tenido } \\
\text { la misma lectura elaborando sintesis común de ideas } \\
\text { principales. } \\
\text { Puesta en común en gran grupo donde cada lectura es } \\
\text { presentada con sus ideas principales. }\end{array}$ \\
\hline
\end{tabular}

Jornadas de Formación e Innovación Docente del Profesorado | № 1 (2018)

(c) Esta obra se distribuye con la licencia Creative Commons Reconocimiento-NoComercial-SinObraDerivada Internacional (CC BY-NC-ND 4.0.) 


\begin{tabular}{|c|c|}
\hline RECAPITULACIÓN & $\begin{array}{l}\text { Sintesis de ideas por parte de las docentes apoyada en } \\
\text { ppt. }\end{array}$ \\
\hline MOTIVACIÓN & $\begin{array}{l}\text { Sesión 2: ¿Cómo conectan con el currículum oficial? } \\
\text { Vídeo introductorio sobre los tipos de rincones. } \\
\text { Presentación del informe }\end{array}$ \\
\hline $\begin{array}{l}\text { AMPLIACIÓN Y } \\
\text { CONTRASTRE }\end{array}$ & $\begin{array}{l}\text { Selección de temáticas del rincón por cada grupo, en base } \\
\text { a un listado dado por las docentes. } \\
\text { Justificación de la temática en base al currículum: } \\
\text { Breve presentación dialogada sobre el marco legal. } \\
\text { Lectura de la Orden que regula el currículum de Ed. } \\
\text { Infantil. } \\
\text { Elaboración y justificación del valor educativo de la temá- } \\
\text { tica elegida en base al marco legal. } \\
\text { Objetivos: } \\
\text { Exposición dialogada sobre la formulación de objetivos. } \\
\text { Definición de los objetivos de cada rincón. }\end{array}$ \\
\hline RECAPITULACIÓN & Puesta en común en gran grupo lo trabajado en la sesión. \\
\hline MOTIVACIÓN & $\begin{array}{l}\text { Sesión 3: ¿Cómo seleccionamos y secuenciamos los } \\
\text { contenidos? } \\
\text { Presentación de recursos y materiales sobre algunas de } \\
\text { las temáticas elegidas }\end{array}$ \\
\hline $\begin{array}{l}\text { AMPLIACIÓN Y } \\
\text { CONTRASTE }\end{array}$ & $\begin{array}{l}\text { Elaboración de un dossier con información sobre el con- } \\
\text { tenido que aborda cada uno de los rincones. Cada grupo } \\
\text { debe informarse sobre la temática que va a trabajar y pre- } \\
\text { parar un dossier con documentación. }\end{array}$ \\
\hline RECAPITULACIÓN & $\begin{array}{l}\text { Sesión } 4 \\
\text { Puesta en común en gran grupo de los dossier elaborados. }\end{array}$ \\
\hline \multirow{2}{*}{$\begin{array}{l}\text { AMPLIACIÓN Y } \\
\text { CONTRASTE }\end{array}$} & $\begin{array}{l}\text { Presentación de mapas de contenidos elaborados por do- } \\
\text { centes y por niños. } \\
\text { Diálogo sobre las posibilidades de los mapas para la se- } \\
\text { lección y secuenciación del contenido. }\end{array}$ \\
\hline & $\begin{array}{l}\text { Sesión } 5 \\
\text { Elaboración de mapas de contenidos para cada uno de los } \\
\text { rincones }\end{array}$ \\
\hline RECAPITULACIÓN & Puesta en común en gran grupo de los mapas elaborados. \\
\hline MOTIVACIÓN & $\begin{array}{l}\text { Sesión 6: ¿Qué tipos de actividades diseñamos en un } \\
\text { rincón? } \\
\text { Realización de actividades libres y dirigidas planteadas } \\
\text { por las docentes }\end{array}$ \\
\hline $\begin{array}{l}\text { AMPLIACIÓN Y } \\
\text { CONTRASTE }\end{array}$ & $\begin{array}{l}\text { Exposición dialogada sobre los tipos de actividades } \\
\text { Diseño de actividades libres y dirigidas para cada rincón }\end{array}$ \\
\hline RECAPITULACIÓN & Puesta en común de las actividades diseñadas \\
\hline
\end{tabular}

Jornadas de Formación e Innovación Docente del Profesorado | № 1 (2018) Esta obra se distribuye con la licencia Creative Commons Reconocimiento-NoComercial-SinObraDerivada Internacional (CC BY-NC-ND 4.0.) 


\begin{tabular}{|c|l|}
\hline MOTIVACIÓN & $\begin{array}{l}\text { Sesión } 7 \\
\text { Debate sobre la evaluación de los rincones }\end{array}$ \\
\hline $\begin{array}{c}\text { AMPLIACIÓN Y } \\
\text { CONTRASTE }\end{array}$ & $\begin{array}{l}\text { Exposición dialogada con apoyo de ejemplos prácticos } \\
\text { para la formulación de criterios de evaluación y el diseño } \\
\text { de instrumentos. } \\
\text { Formulación de criterios de evaluación y diseño de algún } \\
\text { instrumento para cada rincón. }\end{array}$ \\
\hline RECAPITULACIÓN & $\begin{array}{l}\text { Puesta en común de los criterios e instrumentos } \\
\text { elaborados. }\end{array}$ \\
\hline $\begin{array}{c}\text { SÍNTESIS Y CO- } \\
\text { MUNCACIÓN DE } \\
\text { LO APRENDIDO }\end{array}$ & $\begin{array}{l}\text { Sesión } 8 \\
\text { Exposición de los rincones diseñados }\end{array}$ \\
\hline IDEAS FINALES & $\begin{array}{l}\text { Sesión } 9 \\
\text { Valoración global del bloque y recogida de ideas finales }\end{array}$ \\
\hline
\end{tabular}

\section{Secuencia de actividades.}

Para conocer las ideas de los alumnos a lo largo del proceso y poder establecer modelos nos planteamos dos instrumentos:

- Cuestionario Inicial/Final. Es un cuestionario que se cumplimentó de manera individual al inicio y finalización de la experimentación. Constaba de 10 preguntas abiertas que se relacionaban con los tres grandes problemas que planteamos ¿Qué son los rincones y cómo se organiza en un aula de Infantil?, ¿Cómo conectan con el curriculum oficial?, ¿Cómo abordamos los elementos curriculares en el diseño de un rincón? El análisis de los cuestionarios iniciales lo realizamos teniendo en cuenta sus respuestas y organizándolas de menor a mayor grado de complejidad (deseable) en escaleras para ver visualmente cuales son los modelos iniciales y las dificultades de aprendizaje que podamos detectar. Al final del proceso realizamos el mismo análisis para observar su evolución

- Informe Final. Para analizar la evolución del aprendizaje a lo largo del proceso se solicitó a los estudiantes que realicen un informe final de manera grupal. Además este informe formará parte de la evaluación del alumnado en este bloque. 


\section{Aplicación del Ciclo de Mejora Docente}

Primero de todo, debido a la complejidad y amplitud de los contenidos propuestos, la experimentación no se ha terminado tal y como se había propuesto por dos motivos principalmente:

- Se ha tenido que incluir un aspecto que no se contemplaba al inicio en el diseño: ¿Cómo son las ideas del alumnado sobre ciencias y cómo averiguarlo?

- Los problemas-contenidos propuestos no se han ajustado a la temporalización propuesta.

La secuencia transcurrió como estaba previsto hasta la sesión 6, aunque se nos alargó la puesta en común de los mapas de problemas. En este momento vimos la necesidad de conectar la intervención que estábamos realizando con la programación que realizábamos de manera paralela en la asignatura, incorporando las ideas del alumnado. Para desarrollar esta parte comenzamos viendo un vídeo sobre las ideas de los alumnos, en el que se presentan sus principales características y se muestran diversas asambleas llevadas a cabo en aulas de infantil, que permite acercar al alumnado a la realidad del aula. Se ofrecieron algunas pautas sobre cómo diseñar un instrumento para conocer las ideas de los alumnos y comenzamos el diseño. Los instrumentos debían tener claras las preguntas que se hacían y el objetivo que se perseguía con ella, esta es una tarea que hay que concretar bastante porque a veces las preguntas que se realizan son demasiado generales, algunas veces no tienes respuestas claras o no están redactadas para el nivel de los niños y niñas. Posteriormente fuimos a un centro para que pudieran aplicarlo con un grupo real de alumnos. Esta salida resultó muy motivadora, aunque supuso la incorporación de tiempo extra. Tras la recogida de ideas, que fueron grabadas en audio, los estudiantes realizaron un análisis determinando perfiles y estableciendo niveles de complejidad del conocimiento de los niños, al tiempo que identificaban las características del pensamiento infantil. Es justo en este punto, en el que 
nos encontramos cuando estamos redactando esta comunicación, por lo que no hemos podido abordar los contenidos previstos relacionados con el diseño de actividades y la evaluación. Tampoco hemos llegado aún a la exposición de los rincones diseñados. Continuaremos trabajando para lograrlo antes del fin del cuatrimestre.

\section{Evaluación del aprendizaje de los estudiantes}

Para conocer las ideas de los estudiantes a lo largo del proceso nos planteamos dos instrumentos fundamentalmente, un cuestionario inicial-final y un informe final. Como se comentaba en el apartado anterior debido a que no se ha llegado a cerrar la propuesta planteada, para la presentación de resultados iniciales y finales se han podido analizar las seis primeras preguntas del cuestionario. Y por este mismo motivo estamos pendientes de la realización del informe final y cuyos resultados no podemos presentar.

A continuación se presentan las escaleras de aprendizaje para el momento inicial y final de este ciclo de mejora en el que se observan diversas evoluciones dependiendo de la pregunta de análisis:

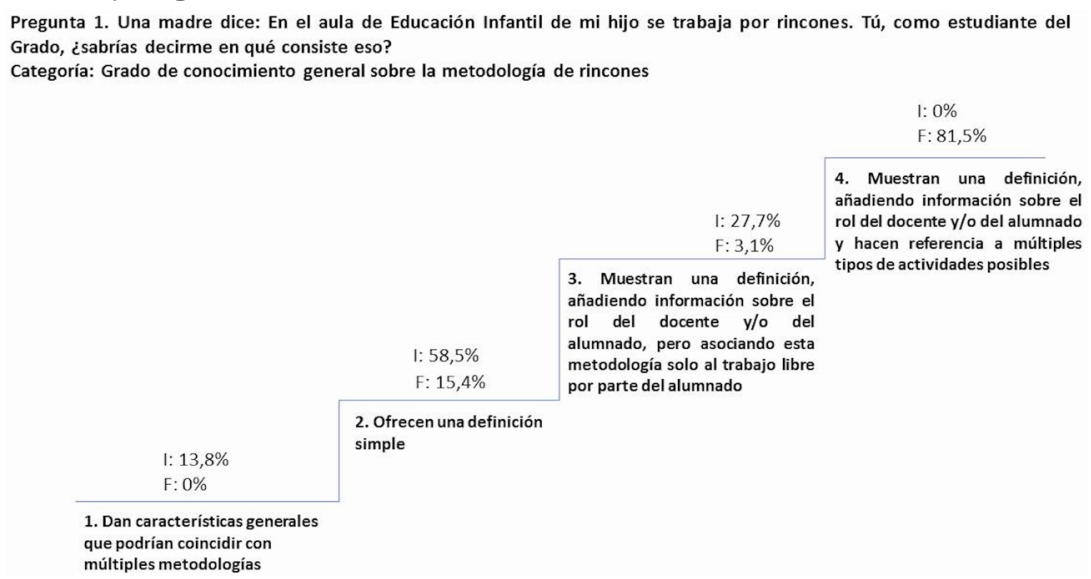

Figura 3. Escalera de aprendizaje sobre la pregunta 1

Jornadas de Formación e Innovación Docente del Profesorado I № 1 (2018) Esta obra se distribuye con la licencia Creative Commons Reconocimiento-NoComercial-SinObraDerivada Internacional (CC BY-NC-ND 4.0.) 
En esta primera escalera podemos apreciar como la idea de los alumnos sobre la metodología de los rincones evoluciona favorablemente tras el CMD, así mientras en el inicio ningún alumno llegaba a dar una respuesta del nivel 4, al final más del 80\% se sitúa en él.

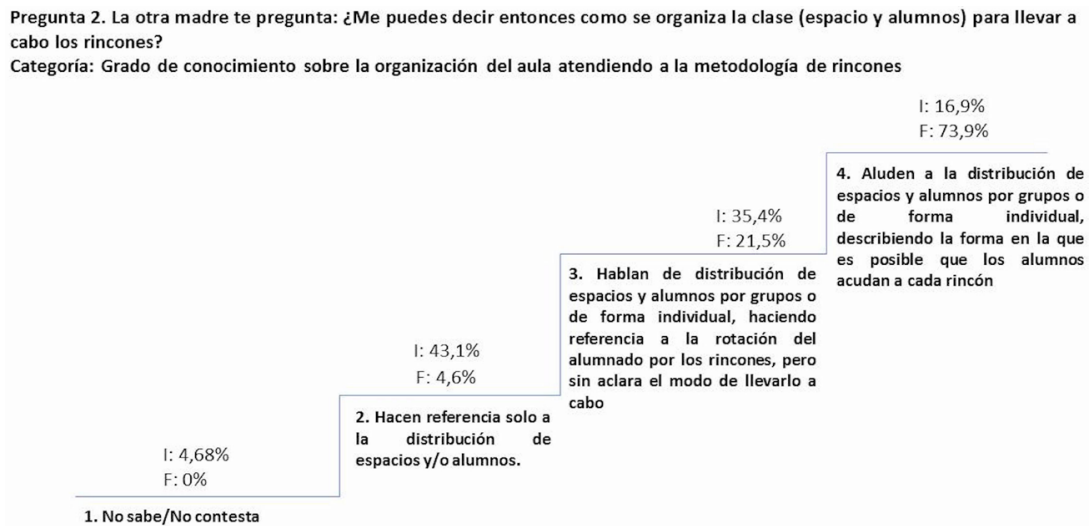

Figura 4. Escalera de aprendizaje sobre la pregunta 2

En la pregunta dos también apreciamos evolución y podemos ver que al inicio los porcentajes más amplios de respuestas se encuentran en los niveles iniciales y al final se localizan en los más elevados.

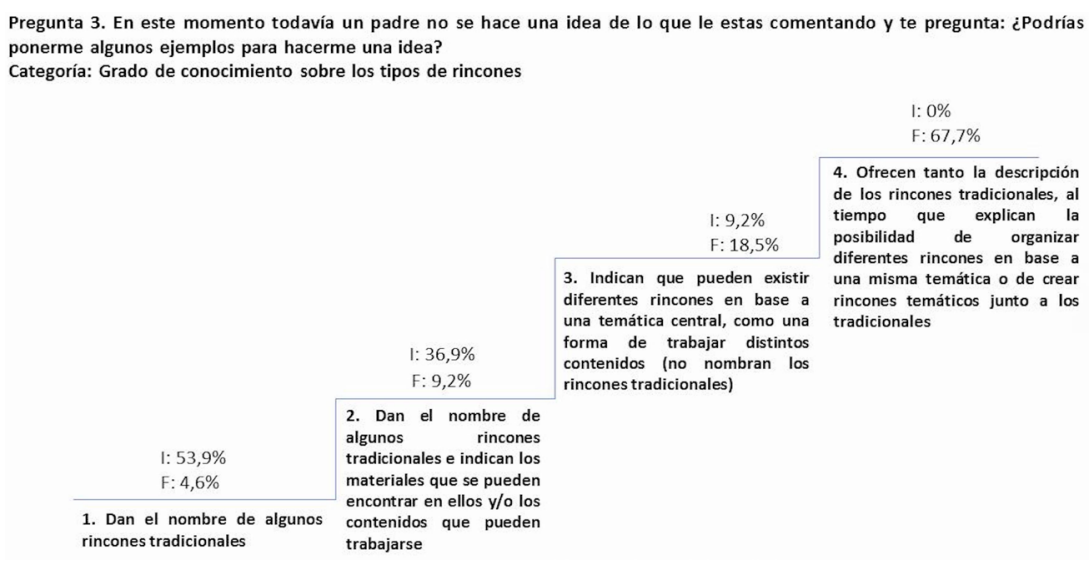

Figura 5. Escalera de aprendizaje sobre la pregunta 3 
Al preguntar por los tipos de rincones, se aprecia que tras el CMD las ideas de los alumnos evolucionan hacia niveles más complejos. En este caso, la evolución es bastante ya que al inicio más del $50 \%$ del alumnado se situaba en el nivel $1 \mathrm{y}$ al final el 67,7\% alcanza el nivel 4 .

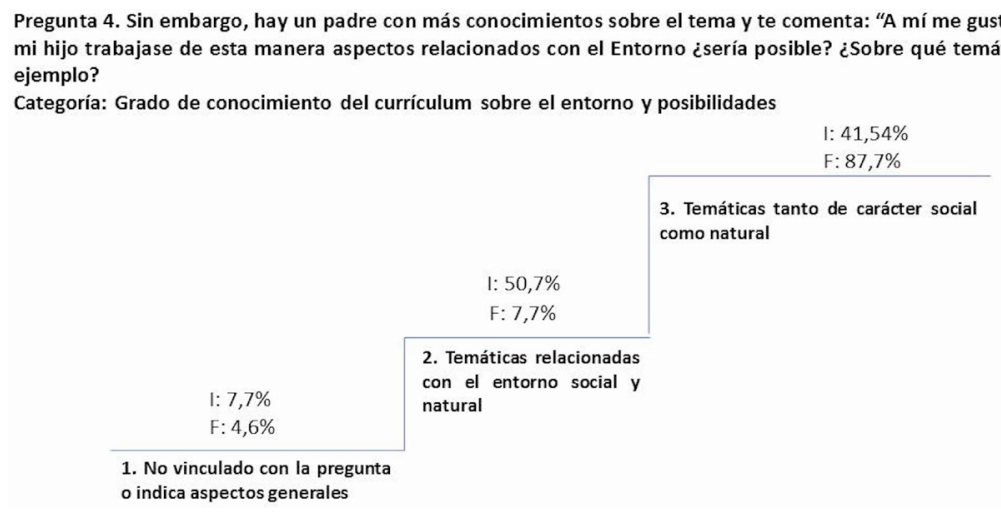

Figura 6. Escalera de aprendizaje sobre la pregunta 4

En el análisis de esta pregunta encontramos en el momento inicial que las temáticas relacionadas con las ciencias naturales $(46,15 \%)$ las identifican mucho más que las de sociales $(4,61 \%)$ debido a que la tradición del uso de los rincones se basa fundamentalmente en actividades más prácticas y que suelen estar más vinculados a temáticas de naturales (las plantas, los animales, la contaminación, los residuos). Una vez realizada la intervención, no se produce esta identificación, aunque hay algunos alumnos que siguen nombrando rincones más vinculados a temáticas de naturales.

Jornadas de Formación e Innovación Docente del Profesorado | № 1 (2018)

(i) Esta obra se distribuye con la licencia Creative Commons Reconocimiento-NoComercial-SinObraDerivada Internacional (CC BY-NC-ND 4.0.) 
Pregunta 5. ¿Se plantean objetivos a la hora de diseñar un rincón? Pon algún ejemplo Categoría: Grado de conocimiento sobre el elemento curricular objetivos

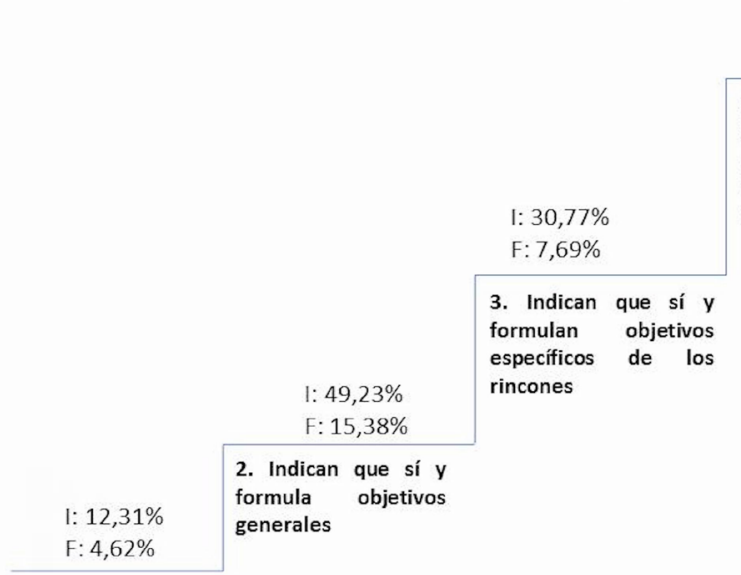

1. Dicen que si pero no

lo justifican

I: $7,69 \%$

$\mathrm{F}: 72,31 \%$

4. Indican que sí y formulan tanto objetivos generales como específicos de los rincones

Figura 7. Escalera de aprendizaje sobre la pregunta 5

Nos damos cuenta en esta 5a pregunta, cómo se plantean en el momento inicial una cantidad de objetivos sin vinculación con los rincones propiamente dichos. Consideramos que es debido a que la redacción de los objetivos se suele hacer de manera muy mecánica sin tener en cuenta el alcance de este elemento a la hora de diseñar un rincón. En el momento final, observamos que ha habido una evolución bastante grande hacia el nivel 3.

Pregunta 6 (primera parte). ¿Qué papel tienen los contenidos?

Categoría: Grado de conocimiento del contenido curricular: contenidos

I: $3,07 \%$

$\mathrm{F}: 38,46 \%$

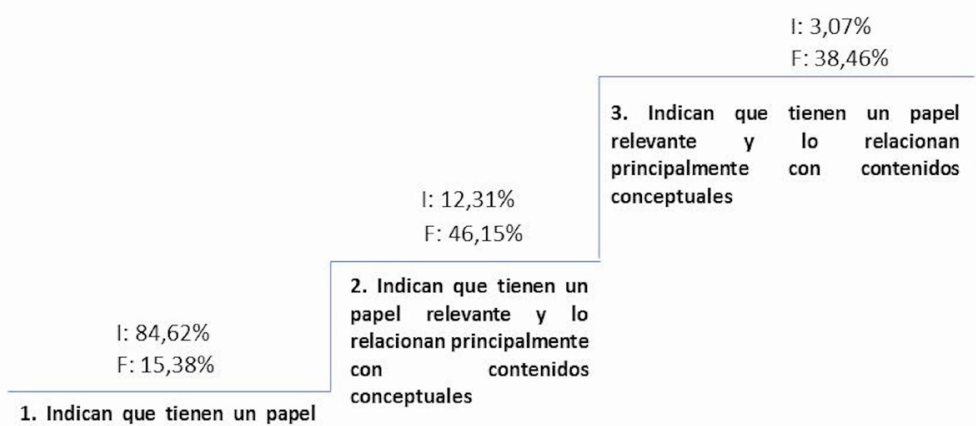

1. Indican que tienen un papel

relevante sin justificación

Jornadas de Formación e Innovación Docente del Profesorado I № 1 (2018)

(c) $\odot$ Esta obra se distribuye con la licencia Creative Commons Reconocimiento-NoComercial-SinObraDerivada 
Pregunta 6 (segunda parte): ¿Quién los propone? ¿Los niños y niñas pueden proponer la temática? Categoría: Papel del alumnado en la selección de los contenidos

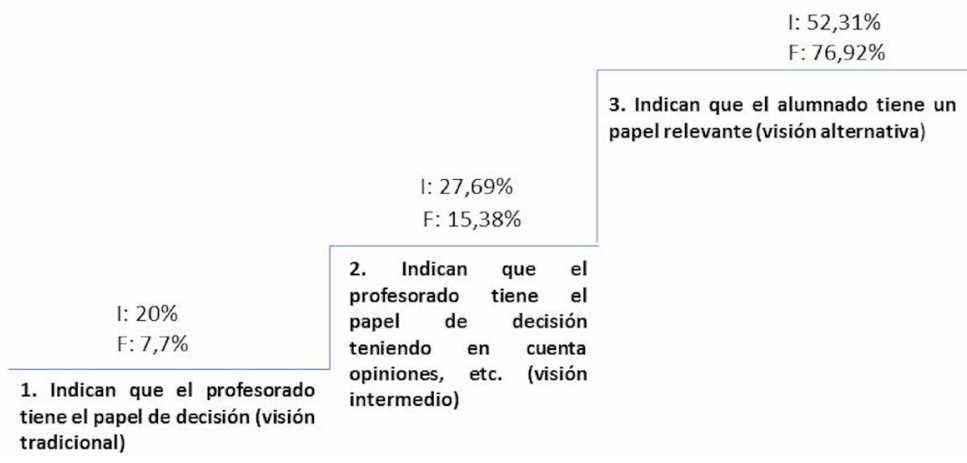

Figuras 8 y 9. Escaleras de aprendizaje sobre la pregunta 6

En relación a la primera parte de la pregunta 6, tenemos que indicar que en el momento inicial tenemos un porcentaje muy alto de respuestas que se posicionan en el valor 1 , y que consideramos que es debido a la redacción poco clara de la pregunta. Por este motivo, en el momento final se reformuló la pregunta indicando ¿qué papel tienen los contenidos? ¿de qué tipo son los contenidos que se pretenden enseñar?. Al realizar este cambio, en el momento final, encontramos respuestas que se posicionan fundamentalmente en el valor 2 y 3.

Respecto a la segunda parte de la pregunta, vemos que tanto en el momento inicial como en el final, el valor 3 está bastante representado, ello es así porque responden desde un punto de vista deseable y consideran a priori el papel del alumnado, aunque pensamos que la puesta en práctica de esto es más complejo y se encontrarán más dificultades.

\section{Evaluación del Ciclo de Mejora Docente}

Analizando los resultados de la evolución del aprendizaje del alumnado, la observación realizada en clase y las reuniones semanales de coordinación con la docente, con 
la que se comparte la propuesta de intervención, hay que destacar que los contenidos planificados son demasiados para el tiempo disponible, además estuvo la necesidad de introducir el subproblema de las ideas del alumnado, para que la propuesta didáctica tuviese un hilo conductor más claro para el alumnado. En este sentido, se considera necesario modificar el mapa de problemas, introduciendo dicho aspecto. Así mismo, en la secuencia de aprendizaje es necesario introducir la secuencia de actividades realizada en la puesta en marcha de este ciclo de mejora y que se comenta en el apartado de relato resumido. A continuación se presenta el mapa de problemas que resultaría tras estas reflexiones.

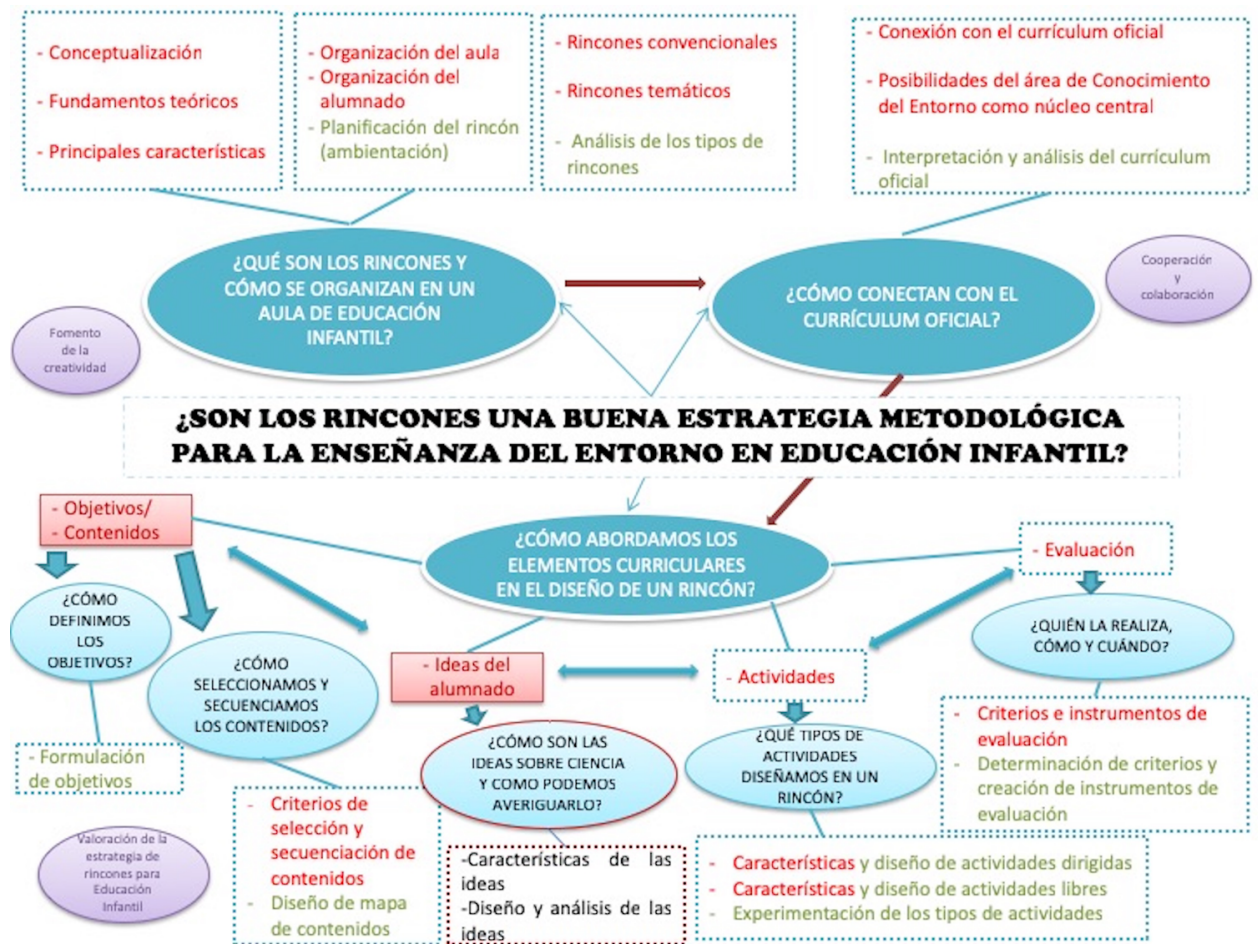

Figura 10. Mapa de contenidos-problemas final

Para finalizar considero que los principios didácticos que han guiado mi experiencia se basan principalmente en el modelo didáctico de referencia o deseable. En este 
sentido el modelo metodológico basado en el principio de investigación (De Alba y Porlán, 2017; García, 2004) ha sido el elegido. Concretando estos principios, puedo indicar que he intentado tener una perspectiva constructivista y evolucionista del conocimiento, una perspectiva compleja del conocimiento que abordo, y una perspectiva crítica.

\section{Referencias bibliográficas}

Ballenilla, F. (2003). El practicum en la Formación Inicial del Profesorado de Ciencias de Enseñanza Secundaria. Estudio de caso. LiberLIBRO.com. Tesis doctoral. Universidad de Sevilla.

De Alba, N. y Porlān, R. (2017). La metodología de enseñanza. En R. Porlán (coord.), Enseñanza Universitaria. Cómo enseñarla (pp.37-51). Madrid: Morata.

García, J.E. (2004): Educación Ambiental, constructivismo y complejidad. Sevilla. Diada Editora.

García-Pérez, F.F (2000). Un modelo didáctico alternativo para transformar la educación: el modelo de investigación en la escuela. Scripta Nova: Revista electrónica de geografia y ciencias sociales, 4, 55-78.

Jornadas de Formación e Innovación Docente del Profesorado | № 1 (2018) Esta obra se distribuye con la licencia Creative Commons 